\title{
Nuclear Pedigree Criteria for the Identification of Individuals Suspected to Be at Risk of an Inherited Predisposition to Gastric Cancer
}

\author{
Beata Gawdis-Wojnarska', Marek Brzosko' ${ }^{2}$ Jacek Fliciński ${ }^{2}$, Krzysztof Marlizz ${ }^{3}$, \\ Teresa Starzyńska ${ }^{3}$, Rodney J. Scott, Jan Lubiński' \\ IInternational Hereditary Cancer Center, Pomeranian Medical University, Szczecin, Poland; 2Clinic of Rheumatology, Pomeranian Medical University, Szzzecin, Poland; \\ ${ }^{3}$ Clinical Gastroenterology, Pomeranian Medical University, Szczecin, Poland; 4Discipline of Medical Genetics, School of Biomedical Sciences, University of Newcastle \\ and the Hunter Medical Research Institute, Australia
}

Key words: hereditary gastric cancer, diagnostic criteria, nuclear families

Corresponding author: Beata Gawdis-Wojnarska, International Hereditary Cancer Center, Pomeranian Medical University, Połabska 4,70-115 Szczecin, Poland. Fax+48914661533, e-mail: bwojnarska@wp.pl

Submitted: 8 March 2004

Accepted: 16 April 2004

\begin{abstract}
Gastric cancer is the second most frequently diagnosed malignancy worldwide and therefore represents a significant healthcare burden. Environmental and genetic factors are involved in the development of gastric cancer. To date only one clear genetic predisposition has been identified involving mutations in the E-cadherin gene. The disease phenotype in patients harbouring E-cadherin mutations appears to be specifically related to diffuse gastric cancer. Little is known genetically about the other forms of gastric cancer. Since there is a growing awareness about the necessity of early intervention criteria have been developed that aid the identification of hereditary forms of gastric cancer. The aim of the current study was to identify minimal inclusion criteria so that nuclear pedigree families can be provided with risk assessment and/or genetic testing.

The results reveal that inclusion features described herein such as (a) gastric cancer diagnosed before 46 years of age; (b) two gastric cancers among first degree relatives diagnosed over the age of 50 are useful in identifying suspected hereditary gastric cancer patients.
\end{abstract}

\section{Introduction}

Gastric cancers represent the second most frequent malignancy worldwide and are often diagnosed at incurable stages [1]. Even though the frequency of gastric cancer is declining in Western countries the global incidence remains relatively high [2]. Currently, there is no clear indication as to why the incidence is declining in Western countries but it is thought that food preservation may play an important role [3]. The causes of stomach cancer are believed to be environmental (such as $H$. pylori infection), genetic or a mixture of both [4]. Notwithstanding, evidence has emerged in recent years that there are definite genetic predispositions to the disease, the most notable being germline mutations in the E-cadherin gene [5]. Mutations in this gene were first identified in 3 Maori kindreds from New Zealand and it was originally believed that E-cadherin mutations were likely to be restricted to similar populations [5]. In subsequent studies $\mathrm{E}$-cadherin mutations were identified in other populations thereby focusing attention on the E-cadherin gene and its role in gastric cancer [6-8]. 
Notably, patients harbouring E-cadherin mutations develop diffuse gastric cancer [9]. Thus far, no E-cadherin mutations have been reported in patients presenting mixed intestinal and diffuse gastric cancer suggesting an alternative genetic predisposition. The genetic basis of intestinal gastric cancer remains to be elucidated and together there may be at least three genes associated with hereditary gastric cancer.

The identification of genetic predispositions to gastric cancer remains a priority since knowledge about the underlying molecular genetic basis of the disease will allow for a better understanding of the mechanisms giving rise to the disease and, perhaps more importantly, allow for the identification of individuals who are at risk of disease development.

At a meeting of the International Gastric Cancer Linkage Consortium a clinical definition of hereditary diffuse gastric cancer and hereditary intestinal gastric cancer was formulated (see a review on gastric cancer by Oliveira et al in this volume). The criteria were designed for all countries except those with a high incidence of disease.

There are two aspects of these criteria that can be problematic in the clinical setting with respect to the identification of familial gastric cancer patients. The first is the difficulty in fulfilling criteria in countries where large families and extensive pedigrees are impossible to be identified, for whatever reason, even though the incidence of hereditary gastric cancer may be quite high. Second, the criteria do not take into consideration the existence of family cancer syndromes where gastric cancer may occur in association with an extra-gastric malignancy.

From a clinical perspective, there is a necessity to be able to identify hereditary stomach cancer families with a minimum set of criteria that will provide a high likelihood of ascertainment. The aim of this study was to determine whether a minimum set of criteria could be established to identify suspected hereditary gastric cancer patients when there is restricted information about the familial occurrence of disease.

\section{Patients and methods}

A total of 198 gastric cancer patients comprising three groups were enrolled in the study.

Group A: Consisted of 60 patients affected by gastric cancer from 28 randomly selected families with at least three gastric cancers among first or second degree relatives, independent of age. The total number of gastric cancer cases identified was 105 but 45 of them were excluded because the cancer family history of respective first degree relatives was not available. All families were registered in the International Hereditary Cancer Center in Szczecin.

Group B: This group comprised 41 patients affected by gastric cancer from 27 randomly selected families with two gastric cancers among first or second degree relatives and at least one of the gastric cancers diagnosed under the age of 50. Similar to Group A, 54 cancer cases were ascertained but 13 had to be excluded because information on the disease in the first degree relatives of the proband could not be retrieved.

Group C: A total of 97 individuals diagnosed with gastric cancer between the years 1993 and 1998 irrespective of family history were collected from the city of Szczecin (total population of 400,000).

The following inclusion criteria for the identification of suspected hereditary forms of gastric cancer were used and compared against one another for their sensitivity and specificity:

Inclusion feature 1: Probands diagnosed with gastric cancer at or under the age of 45, no malignancy of any kind in parents or siblings

Inclusion feature 2: Two gastric cancers among first degree relatives diagnosed over the age of 50

Inclusion feature 3: A first degree relative affected by an "extra-gastric" malignancy at any age

Inclusion feature 4: Gastric cancer diagnosed under the age of 46 and a first degree relative affected by an "extra-gastric" malignancy diagnosed at any age.

\section{Statistical analysis}

Univariate statistical analysis (Chi-squared, odds ratio $(O R))$, and sensitivity and specificity of selection were performed using the SAS and LOGIT programs.

\section{Results}

The comparison of the four criteria was undertaken from the three patient groups to identify the most consistent criteria that can be employed in the clinical setting for the identification of suspected hereditary gastric cancer from nuclear pedigree data. The first comparison was made between Group A (associated with a genetic predisposition to the disease) and the unselected cases from Group C. The results indicate that if the inclusion feature 2 was applied, a high degree of accuracy was matched between the two groups. On the contrary, inclusion feature 3 also was useful in exclusion of an inherited predisposition to the disease. 
Table 1. Comparison of frequency of analysed clinical features between studied Group A and controls

\begin{tabular}{lccccccc}
\hline Criteria & Group A $(n=60)$ & Group $C(n=97)$ & OR & Cl & Sensitivity & Specificity & $P$ \\
\hline IF1 & 0 & 3 & 0.32 & $0.01-6.73$ & 0.83 & 97.42 & 0.4380 \\
\hline IF2 & 41 & 12 & 15.29 & $6.78-34.47$ & 68.33 & 87.63 & 0.0000 \\
\hline IF3 & 1 & 13 & 0.11 & $0.01-0.86$ & 0.83 & 86.60 & 0.0122 \\
\hline IF4 & 0 & 3 & 0.32 & $0.01-6.73$ & 6.73 & 97.42 & 0.4380 \\
\hline
\end{tabular}

In comparing Group B against Group C inclusion feature 2 was omitted. Nevertheless, using a similar approach, inclusion feature 1 was valuable in identifying similar features in Group C. No other associations were identified.

\section{Discussion}

The recognition of features that can be used for the identification of familial forms of gastric cancer in situations where extensive pedigree analysis is unknown or impossible to ascertain but the prevalence of the disease is relatively high will aid the identification of individuals at increased risk of developing gastric cancer.

By using the criteria described herein and the consequent recognition of significant odds ratios for some of the inclusion features to identify gastric cancer families we believe that the identification of additional genes associated with this malignancy will be expedited.

Of particular interest are the odds ratio values for the inclusion features IF2 between Groups A and C and IF1 between Groups B and C, which were relatively high (15.29 and 10.1, respectively). Since these inclusion features are significant we have a relatively high degree of confidence that the reported observations are not biased and are an accurate reflection of validity of our approach for the identification of hereditary gastric cancer families. Indeed, these criteria have been tested in our outpatient clinics to successfully identify hereditary gastric cancer. Therefore if we have families matching IF1 or IF2 we are confident that a diagnosis of familial gastric cancer can be made with high probability.
At present it seems reasonable to offer an option of annual gastroscopies to all individuals identified with the use of our inclusion features beginning at an age 5 to 10 years younger than the youngest gastric cancer identified within the patient's family. Such surveillance should only be an option and not a recommendation because the efficiency of such management procedures have not been rigorously determined to reduce morbidity and/or mortality. Most probably the real value of surveillance and of potential chemoprevention will have to be established by studies in large cohorts of individuals from families matching pedigree and clinical criteria of suspected hereditary gastric cancer with identified constitutional DNA variants associated with genetic predispositions.

So far, the list of such changes is very limited but should be extended in the near future as more knowledge is gained about the genetic factors associated with altered gastric cancer predisposition.

In summary, we advocate the use of our criteria of suspected hereditary gastric cancer identified in this report in order to:

a. offer a gastroscopy option,

b. create repositories of nuclear gastric cancer families for future studies on the efficiency of surveillance and chemoprevention protocols for individuals with genetic predispositions to gastric cancer.

\section{References}

1. Boyle P. Global cancer burden. Lancet 1997; 349: 23-26.

2. Howson CP, Hiyama T and Wyinder EL. The decline in gastric cancer: epidemiology of an unplanned triumph. Epidemiol Rev 1986; 8: 1-27.

Table 2. Comparison of frequency of analysed clinical features between studied Group $C$ and controls

\begin{tabular}{lccccccc}
\hline Criteria & Group B $(n=41)$ & Group $C(n=97)$ & OR & Cl & Sensitivity & Specificity & $P$ \\
\hline IF1 & 10 & 3 & 10.1 & $2.6-39.1$ & 24.4 & 96.9 & 0.0001 \\
\hline IF3 & 7 & 13 & 1.3 & $0.5-3.6$ & 17.1 & 86.6 & 0.5756 \\
\hline IF4 & 1 & 3 & 0.8 & $0.1-7.8$ & 7.8 & 96.9 & 0.8343 \\
\hline
\end{tabular}


3. Fuchs CS and Mayer RJ. Gastric carcinoma. N Engl J Med 1995; 333: 32-41.

4. Parsonnet J, Hansen S, Rodriguez L, Gelb AB, Warnke RA, Jellum $E$, Orentreich N, Vogelman JH and Friedman GD. Helicobacter pylori infection and gastric lymphoma. N Engl J Med 1994; 330 (18): 1267-1271.

5. Guilford P, Hopkins J, Harraway J, McLeod M, McLeod N, Harawira P, Taite H, Scoular R, Miller A and Reeve AE. E-cadherin germline mutations in familial gastric cancer. Nature 1998; 392: 402-405.

6. Gayther SA, Gorringe KL, Ramus SJ, Huntsman D, Roviello F, Grehan N, Machado JC, Pinto E, Seruca R, Halling K, MacLeod P, Powell SM, Jackson CE, Ponder BA and Caldas C. Identification of germ-line $\mathrm{E}$-cadherin mutations in gastric cancer families of European origin. Cancer Res 1998; 58: 4086-4089.

7. Richards FM, McKee SA, Rajpar MH, Cole TR, Evans DG, Jankowski JA, McKeown C, Sanders DS and Maher ER. Germline $\mathrm{E}$-cadherin gene $(\mathrm{CDH} 1)$ mutations predispose to familial gastric cancer and colorectal cancer. Hum Mol Genet 1999; 8: 607 . 610.

8. Zhu ZG, Yu YY, Zhang Y, Ji J, Zhang J, Liu BY, Chen XH, Lu Y, Jiang $H S, B u L, H u L D$ and Kong $X Y$. Germline mutational analysis of $\mathrm{CDH} 1$ and pathologic features in familial cancer syndrome with diffuse gastric cancer/breast cancer proband in a Chinese family. Eur J Surg Oncol 2004; 30: 531-535.

9. Caldas C, Carneiro F, Lynch HT, Yokota J, Wiesner GL, Powell SM, Lewis FR, Huntsman DG, Pharoah PD, Jankowski JA, MacLeod P, Vogelsang H, Keller G, Park KG, Richards FM, Maher ER, Gayther SA, Oliveira C, Grehan N, Wight D, Seruca R, Roviello F, Ponder BA and Jackson CE. Familial gastric cancer: overview and guidelines for management. J Med Genet 1999; 36: $873-880$ 